\title{
A Brief Review of the High-order Numerical Manifold Method
}

\author{
H.H. Zhang ${ }^{1, a}$, X.L. Ji ${ }^{1, b}$, \\ ${ }^{1}$ School of Civil Engineering and Architecture, Nanchang Hangkong University, Nanchang, Jiangxi, \\ 330063, PR China \\ ahhzhang@nchu.edu.cn, ${ }^{b} x \mathrm{lj} 109 @ 163 . c o m$
}

Keywords: Numerical manifold method, High-order approximation, Review

Abstract: The numerical manifold method (NMM) is first proposed by Dr. Shi. The finite cover technology from the mathematical manifold analysis is incorporated into the NMM. Due to the independence of the mathematical cover system from the physical domain, the NMM has unique advantages on the analysis of both continuous and discontinuous problems. In the past decades, extensive effects have been put on the developments and applications of the NMM in different areas. In this paper, special attention was paid to the advances of the higher-order NMM.

\section{Introduction}

Proposed by Shi in 1991, the numerical manifold method (NMM) [1] combines the advantages of the finite element method (FEM) and the discontinuous deformation analysis (DDA), which makes it powerful to uniformly treat both continuous and discontinuous problems. In other words, the FEM and DDA are the special cases of the NMM.

In the NMM, the partition of unity (PU) [2] weight functions and the cover functions are pasted together to approximate the concerned physical fields. The PU weight functions are defined on the mathematical covers, while the cover functions are constructed on the physical covers [1]. The style of the cover functions can be flexibly chosen according to the physical features, e.g., polynomials are frequently used for continuous problems while crack tip asymptotic basis can be further adopted for fracture analysis. Usually, the NMM with constant cover functions is called the conventional NMM (CNMM), while that using higher-order polynomials is termed as the high-order NMM (HONMM). In the recent years, the HONMM has been developed and applied in different fields. In the present paper, the state-of-art of the HONMM is briefly summarized.

\section{General description of the HONMM}

In the NMM, the displacement approximation is generally expressed as

$$
\mathbf{u}^{h}(\mathbf{x})=\sum_{i=1}^{\mathrm{NP}} w_{i}(\mathbf{x}) \mathbf{u}_{i}(\mathbf{x})
$$

where NP is the number of physical covers, $\mathbf{x}=(x, y)$ and $w_{i}(\mathbf{x})$ is the weight function corresponding to the $i$ th physical cover. $\mathbf{u}_{i}(\mathbf{x})$ is the cover function defined on the $i$ th physical cover. For continuous problems, $\mathbf{u}_{i}(\mathbf{x})$ is usually chosen as [3] 


$$
\mathbf{u}_{i}(\mathbf{x})=\mathbf{P}^{\mathbf{T}}(\mathbf{x}) \mathbf{a}_{i}
$$

where $\mathbf{a}_{i}$ is

the vector of degrees of freedom (DOFs) and $\mathbf{P}^{\mathrm{T}}(\mathbf{x})$ is the matrix of polynomial bases. For two-dimensional (2D) problems,

$$
\mathbf{P}^{\mathbf{T}}(\mathbf{x})=\left[\begin{array}{llllllll}
1 & 0 & x & 0 & y & 0 & \mathrm{~L} & 0 \\
0 & 1 & 0 & x & 0 & y & 0 & \mathrm{~L}
\end{array}\right]
$$

As already described in the Introduction part, for the case when $\mathbf{P}^{\mathrm{T}}(\mathbf{x})=\left[\begin{array}{ll}1 & 0 \\ 0 & 1\end{array}\right]$, the NMM is just the CNMM; otherwise, it is named the HONMM.

\section{Some advances of the HONMM}

Since the advent of the NMM, a lot of work has been done on the HONMM from both theoretical and application aspects. To the authors' knowledge, although formulated in the work of Shi [1], the earliest application of the HONMM is carried out by Chen and his co-authors [4]. In their study, the first-order polynomial is taken as the cover function together with the triangular elements. Numerical test on a simply-supported beam shows that the accuracy of the HONMM is much better than that by the CNMM, compared with the analytical solutions.

Cai and Zhang [5] focused on the selection of weight functions and displacement approximations, and also the treatment of the essential boundary conditions in the HONMM. Numerical results verify that the proposed approach is accurate and efficient.

$\mathrm{Ju}$ et al [6] combined the P-adaptive analysis with the NMM on fixed triangular meshes. The associated displacement functions and stiffness matrix are derived. Numerical example shows that the P-adaptive algorithm (HONMM, namely) could improve the accuracy and efficiency as well.

Tian et al [7] derived the general mathematical formulations of the HONMM and developed the associated $\mathrm{C}++$ codes using the first-order and second-order polynomial cover functions. The study on a purely-bending beam demonstrates the excellent agreement between the results by the second-order NMM and the analytical solutions.

Song and Ohnishi [8] developed the HONMM on rectangular mathematical elements. The stress and strain are calculated with the simplex integration. The analysis of cantilever beam shows the high accuracy of the proposed method.

Wang et al [9] used the first-order physical cover function to simulate the crack growth problem on triangular elements. They put forward a scheme to tackle the case when crack tip terminates interior an element, and the corresponding shortcoming in the CNMM for crack propagation is cured.

Using the mathematical software Matematica, Su et al [10] automatically derived the equations and generated codes of the HONMM. The results of typical cases indicate that the numerical solutions are improved with the increase of the order of cover functions.

He et al [11] applied the HONMM to study the mechanical behavior of beam and truss in underground structure. As shown in numerical calculations, the proposed beam element is accurate and effective.

Liu and Yang [12] introduced the HONMM to model Hopkinson dynamics ruptures. The general formulates are derived and the failure process of a rock bar is computationally reproduced. 
Lin et al [13] adopted special matrix operations, e.g., the Kronecker product and Hadamard product, to ease the derivation of element matrices of the HONMM. The corresponding three-dimensional (3D) program for elastostatic analysis is developed and verified through numerical examples.

Jiang et al [14] developed the 3D HONMM. The first-order cover function is used to calculate the subsidiary stress in rock mechanics and the corresponding results are much closer to analytical solutions compared with those by the CNMM.

To find a balance between the accuracy and efficiency of solution, Deng et al [15] put forward a mixed strategy, that is, the low-order cover functions are applied to analyze simple deformation region while the higher-order ones are used in the complex zone. The reliability and effectiveness of the proposed scheme is validated through a typical geotechnical example.

Kourepinis et al [16] analyzed the fracture of concrete structures with the HONMM. The high-order approximation is realized by hierarchically increasing the order of displacement polynomials.

Su et al [17] derived the initial stress equation in the HONMM to reflect configuration change in large deformation problems. The validity of the equation is proved in finite deformation analysis of a cantilever beam.

Liu and Chen [18] studied the viscoelastic deformation of rock mass with the HONMM. The "time-initial strain" method is applied to consider the creep behavior. Compared with the CNMM, the accuracy is improved with the present approach.

Guo and Zheng [19] focused on the linear dependence (LD) problem [20, 21] in the HONMM. An improved LDLT algorithm is developed to obtain a particular solution to the linear equations of the HONMM. It is found that both the accuracy and efficiency of the approach is superior to some frequently used methods (e.g., the perturbation method).

Ghasemzadeh et al [22] used the HONMM and the weighted residual method to solve 2D dynamics problems. A modified Newmark integration is applied for time integration of high-order equations and the LD problem is also solved by adjusting the simulation functions.

To tackle the LD problem in the HONMM, Xu et al [23] adopted the first-order Taylor's expansions as the cover functions, where the DOFs bear definite physical meanings. In addition, they also discussed the choice of physical cover type around the boundary.

Using the first-order Taylor's expansions and crack tip asymptotic basis in the cover functions, $\mathrm{Xu}$ et al [24] captured 2D crack growth path by the HONMM. Furthermore, a physical cover updating scheme is given for both small and large deformation problems.

Liu et al [25] combined the HONMM with the hexahedron element to solve 3D rock mechanics problems. The modified LDLT scheme presented in [19] is adopted to tackle LD problem.

Fan et al [26] added the nine-node triangular element to the NMM. The LD problem is avoided through the use of third-order weight functions together with constant local approximation. Although higher-order approximation is also realized, their idea is different from our defined HONMM.

Based on the work in [23] and [24], $\mathrm{Xu}$ et al [27] further presented a linearly independent HONMM for 2D crack problems. Numerical tests show that the LD trouble is totally avoided and the fracture parameters, i.e., the stress intensity factors, agree well with reference solutions.

Considering the advantages of polygonal elements, Ji [28] studied the LD problem and the accuracy of the HONMM on hexagonal elements. It is found that the LD problem also arises in 
polygonal HONMM and the accuracy of the proposed method depends on the exact solution of physical examples.

\section{Conclusions and discussion}

Due to its high accuracy and acceptable computational cost, the high-order numerical manifold method is widely studied in the past years. In this paper, the recent developments of the HONMM are briefly reviewed, from both theoretical and engineering aspects. Although a lot of work has been carried out, the HONMM is still young. For further improvements and applications of the HONMM, in the authors' view, special attention may be paid to the following two topics:

(1) The LD problem is still a bottleneck of the HONMM, although some studies have been carried out, e.g., in [19-23, 25-28]. The solution to the LD problem in both 2D and 3D cases with much more elegant and universal scheme is still open.

(2) The use of high-order cover functions may increase the numerical cost. How to increase the efficiency is also an attractive issue.

\section{Acknowledgements}

The present work was supported by the National Natural Science Foundation of China (Grant No. 11462014), the Provincial Natural Science Foundation of Jiangxi, China (Grant No. 20151BAB202003) and the Science and Technology Program of Educational Committee of Jiangxi Province of China (Grant No. GJJ14526).

\section{Reference}

[1] G.H. Shi, Manifold method of material analysis, in: Transaction of 9th Army Conference on Applied Mathematics and Computing, Minneapolis, Minnesota (1991) 57-76.

[2] J.M. Melenk, I. Babuska, The partition of unity finite element method: basic theory and applications, Comput. Methods Appl. Mech. Eng. 139 (1996) 289-314.

[3] H.H. Zhang, L.X. Li, X.M. An, et al, Numerical analysis of 2-D crack propagation problems using the numerical manifold method, Eng. Anal. Bound. Elem. 34 (2010) 41-50.

[4] G.Q. Chen, Y. Ohnishi, I. Tkahiro, Development of high-order manifold method, Int. J. Numer. Meth. Eng. 43 (1998) 685-712.

[5] Y.C. Cai, X.W. Zhang, Expansion to high-order cover function and improvement of the stress accuracy in numerical manifold method, Chinese Journal of Mechanical Engineering 36 (2000) 20-25.

[6] Y.F. Ju, G. Zhang, S.L.Wang, A preliminary study of numerical manifold method in P-adaptive analysis, Rock and Soil Mechanics 22 (2001) 88-91 (in Chinese).

[7] R. Tian, M.T. Luan, Q. Yang, et al, Fundamentals and applications of high-order manifold method, Engineering Mechanics 18 (2001) 21-26 (in Chinese).

[8] J.S. Song, Y. Ohnishi, High order rectangular element of manifold method, Chinese Journal of Rock Mechanics and Engineering 22 (2003) 932-936.

[9] S.L. Wang, X.T. Feng, X.R. Ge, Study on crack propagation modeling by high order manifold method, Rock and Soil Mechanics 24 (2003) 622-625 (in Chinese).

[10]H.D. Su, X.L. Xie, Q. Chen, Application of high-order numerical manifold method in static analysis, Journal of Yangtze River Scientific Research Institute 22 (2005) 74-77, 91 (in Chinese). 
[11] Y.P. He, J. Huang, H.H. Zhu, et al, Computation of beam and truss for underground structure using high-order manifold method, Chinese Journal of Underground Space and Engineering 1 (2005) 674-678, 684.

[12]H.Y. Liu, J. Yang, Simulation of Hopkinson dynamic ruptures experiment by high-order numerical manifold method, Journal of China Coal Society 30 (2005) 340-343.

[13] S.Z. Lin, Y.F. Qi, H.D. Su, Element analysis of high-order numerical manifold method based on special matrix operations, Journal of Yangtze River Scientific Research Institute 23 (2006) 36-39 (in Chinese).

[14]Q.H. Jiang, S.S. Deng, C.B. Zhou, Study of three-dimensional high-order numerical manifold method, Rock and Soil Mechanics 27 (2006) 1471-1474 (in Chinese).

[15]A.F. Deng, A.J. Zhu, X.Y. Zeng, The numerical manifold method using low-order and high-order cover functions, China Civil Engineering Journal 39 (2006) 75-78.

[16]D. Kourepinis, C. Pearce, N. Bicanic, Higher-order discontinuous modeling of fracture in concrete using the numerical manifold method, Int. J. Comp. Meth. 7 (2010) 83-106.

[17]H.D. Su, J.H. Cui, X.L. Xie, Initial stress equation for high-order numerical manifold method, Chinese Journal of Computational Mechanics 27 (2010) 270-274.

[18] J. Liu, Q. Chen, Study of high-order numerical manifold method in viscoelastic creep of rock mass, Rock and Soil mechanics 33 (2012) 2174-2180 (in Chinese).

[19]C.X. Guo, H. Zheng, Study on linear dependence problem in high-order numerical manifold method, Engineering Mechanics 29 (2012) 228-232 (in Chinese).

[20]X.M. An, L.X. Li, G.W. Ma, et al, Prediction of rank deficiency in partition of unity-based methods with plane triangular or quadrilateral meshes, Comput. Methods Appl. Mech. Eng. 200 (2011) 665-674.

[21]X.M. An, Z.Y. Zhao. H.H. Zhang, et al, Investigation of linear dependence problem of three-dimensional partition of unity-based finite element methods, Comput. Methods Appl. Mech. Eng. 233-236 (2012) 137-151.

[22]H. Ghasemzadeh, M. A. Ramezanpour, S. Bodaghpour, Dynamic high order numerical manifold method based on weighted residual method, Int. J. Numer. Meth. Eng. 100 (2014) 596-619.

[23]D.D. Xu, H. Zheng, Y.T. Yang, Linearly independent higher-order numerical manifold method, Chinese Journal of Geotechnical Engineering, 36 (2014) 482-488.

[24]D.D. Xu, H. Zheng, K.W. Xia, et al, Application of higher-order enriched numerical manifold method to crack propagation, Chinese Journal of Rock Mechanics and Engineering 33 (2014) 1375-1387.

[25]D.X. Liu, Y.L. Zhang, F. Tan, et al, Discussion on high-order numerical manifold method based on hexahedron element, Rock and Soil Mechanics 35 S2 (2014) 662-666, 675 (in Chinese).

[26]H. Fan, S.M. He, Z.M. Jiang, A high-order numerical manifold method with nine-node triangular meshes, Eng. Anal. Bound. Elem. 61 (2015) 172-182.

[27]D.D. Xu, Y.T. Yang, H. Zheng, et al, Application of the linearly independent high-order numerical manifold method in the fracture mechanics, Chinese Journal of Rock Mechanics and Engineering 34 (2015) in press.

[28]X.L. Ji, The high-order polygonal numerical manifold method and its application in linear elastostatic, Master's thesis, Nanchang Hangkong University, Nanchang, 2015 (in Chinese). 